\title{
Clinical Pharmacist in Nuclear Medicine: A New Role in Ascendancy
}

\author{
Jyoti Patel and Sandipkumar Bhatt* \\ Department of Pharmacology \& Clinical Pharmacy, K.B. Institute of Pharmaceutical Education and Research, Gh-6, Sector-23, \\ Gandhinagar, Gujarat
}

\begin{abstract}
Nuclear pharmacy is a unique branch of pharmacy services that focuses on the proper, safe management and use of radiopharmaceuticals. Most of pharmacist spent their time in traditional services while few renders clinical services in hospital setting even recruitment and regulatory body also demand undergraduate qualification which may find insufficient to render services. It is a matter of question how many provides clinical or pharmaceutical care? But spending a time in clinical activities by pharmacist is an issue of debate. After satisfactory completion of project in this area, attempt has been made by this article to explore our perception on scope, education and functions of pharmacist especially by hospital and by clinical pharmacist. How pharmacist can perform pharmaceutical care in this unique service area is discussed and responsibilities of pharmacist in nuclear pharmacist are proposed.
\end{abstract}

Keywords: Nuclear medicine, clinical pharmacy, radiations, pharmaceutical care.

\section{INTRODUCTION}

What is the application of radiopharmaceuticals? What is use of lead shield? What is barium meal and how 99Tc works? What are its applications? Why do we study? For the time being, questions were solved from standard texts but really we explore and understand it's application when we completed our project in radio and nuclear pharmacy at Nuclear Medicine department at Gujarat Cancer Research Society, Ahmadabad. From its starting, pharmacy has been recognized as pious health care professional. Last century witnessed marvelous development in various field. During 1950's and 60's of last century, voluminous inventions were carried out. Of the all especially in radio and nuclear pharmacy is 99Tc generator. There was an urgent need of someone who works in this area to balance the need of such rapid scientific development were on the way. It was become apparent that radio pharmacist was very definitely needed in major department of many hospitals in developed country especially at USA. To make pharmacist enough competent training was given and later certificate course, special course and now strict laid down criteria along with education come in force with time to time. Parallel, pharmacy has also expanded their services and filled vacuum and void and hence baring out as a special designation cum service as a nuclear pharmacist. Nuclear pharmacy is- also referred as radio pharmacy at many places is now a well recognized and credible specialty. Nuclear pharmacy uses the radiopharmaceuticals needless to say for diagnosis and least for treatment of diseases and thus, improves and promotes health. Each country has their own rules and regulations concerned to pharmaceuticals. Regulatory
Submitted Date : 09-06-2014 Accepted Date : 31-08-2014

DOI: $10.5530 /$ ijopp.7.3.4

Address for correspondence: Bhatt SP

Department of Pharmacology \& Clinical Pharmacy, K.B. Institute of Pharmaceutical Education and Research, Gh-6, Sector-23, Gandhinagar, Gujarat. 382023

Phone: 079-232-49069

E-mail: sunrisedeep78@ rediffmail.com

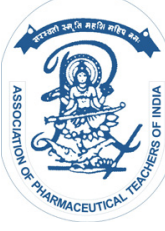

www.ijopp.org 
body of concerned country decides role, responsibilities of personnel dealing with pharmaceuticals. It true for radiopharmaceuticals too. In west, especially at USA, concept of radio pharmacy was started back by 1955 and education was started in between 1968-70. The history, education at USA and development over all are discussed in many of textbook published by renowned Indian authors and reviewed in more recently published article in journal. ${ }^{1}$ Concept and applied knowledge of radio and nuclear pharmacy is remained bound in text rather then applied. Education, role and responsibilities of nuclear pharmacist are well defined at overseas but this is not true in case of India. The central point is specialized training, education, training and working environment in/on routine basis is essential to prepare nuclear pharmacist rather than recruiting pharmacist to perform traditional functions. After successful initiation of Pharm D. in India, clinical pharmacy is in forwarding stage. ${ }^{2}$ Entire practicing clinical pharmacy professionals are presently pay attention and consumed in efforts to identify ways that pharmacist can have a beneficial impact on the welfare of the patients. This challenge of rendering clinical and pharmaceutical services is also a point of attention to policy makers in radio pharmacy too. Traditional function of pharmacist are needlessly performed by pharmacist and technicians but current nuclear pharmacy services and practice, irrespective of working domain, continues to revolve around the radiopharmaceutical product, especially preparation, quality control testing, packaging, storage, distribution and dispensing. Therefore, attempt has been made to explore radio and nuclear pharmacy.

\section{SCOPE \& EMPLOYMENT}

The increasing complexity in health care has created opportunity for pharmacist in many practice setting. Today, pharmacist expanded their services from its traditional services. Clearly, the opportunities that pharmacist grabs and evolves as health care provider.

In India, pharmacy practice and service in radiopharmaceutical as radio pharmacy occurs principally in two settings:

1) Within the nuclear medicine department/settings/ clinic and

2) Within a regulatory and controlling body-Bhabha Atomic Research Centre (BARC, AERB) in India.

Last year, BARC announce recruitment of pharmacist B. ${ }^{3}$ In order to characterized the nature of services and function it rotate towards radiopharmaceuticals. It is traditional and distributive functions. Once applicant qualifies, he/she will undergo training and then work independently under team leader/senior. While at over- seas, especially at USA, beyond above nuclear pharmacy is an independent and unique post after suitable study, training and exposure. ${ }^{4}$ Becoming an authorized nuclear pharmacist is not a easy task. It requires hours of training in nuclear pharmacy, including classroom teaching learning hours and many hours of on-the-job training hours. After exclusive hours of practicing nuclear pharmacy and passing the Board of Pharmacy Specialties (BPS) certification exam, a pharmacist may become a Board Certified Nuclear Pharmacist. Career and working in nuclear pharmacy is a dream, dear and near to many of pharmacist at overseas.

Nuclear pharmacy provides an exciting, challenging, and rewarding environment for pharmacy practice. It allows to learn and applied use a lot of the physics, chemistry and clinical knowledge plus there is a lot of interaction with the expert from different area and health care team.

There are only few articles are published on job satisfaction in this area. Few surveys have been reported and suggest that nuclear pharmacist is satisfied with their jobs. ${ }^{5,6}$ It is important worth noting; how many published article from India? Pharmacist "B" to Pharmacist "F" exists in BARC with prescribed scale. Remuneration and category of Pharmacist offered by BARC in last advertisement may become an issue of debate. Because, applicant required was with diploma qualification and able to perform traditional distributive services and under the category "TECH". Of the 899 applicants for the post Pharmacist B, 31 were invited for trade test and interview. ${ }^{7}$

A how many pharmacists are rendering clinical service at nuclear medicine department and how many employed in BARC will be the cue for new opening. At present, pharmacy practice is in developing stage, therefore, pharmacists' scope is more valuable and must increase capacity to render the service as revolutionary change in nuclear pharmacy. Pharmacist working in nuclear medicines has unique personality, skill, characters and thus reflects a high degree of professionalism as compared to other pharmaceutical service.

\section{CLINICAL PHARMACY SERVICES IN NUCLEAR PHARMACY}

Clinical pharmacy is well defined in its services not only in literature but also in practice. As written earlier clinical pharmacy in India is in developing stage therefore question may arise that what are the clinical services possible in nuclear medicine. Pharmacist in this specialty can render services related to medicine management and clinical services. Medicine management and distribution is traditional functions while clinical activity includes pharmaceutical care, patient education, patient 
counseling, patient history taking, medication chart review, medication order review, intervention on drug related problem and error reporting. One of the unique clinical activity - ward round participation is impossible but discussion among health care team members is possible. Plethora of journal published articles on successful patient care by collaborative work performed by physician, nurse, technicians and pharmacist in professional manner rather than submissive way. ${ }^{8,9,10,11}$ The schematic diagram in figure.1 illustrates working of clinical pharmacist within healthcare at nuclear medicine department.
There are two main functions pharmacist can perform in present time; one is counseling- past medicine history interview \& counseling, second is pharmaceutical care. The pharmacist in radio and nuclear pharmacy may be the most appropriate position to counsel and educate patients by the education and experience. Unawareness of patients on application and use of radio pharmaceuticals lead to total failure of procedure. Patients are benefited by providing proper counseling on use of radiopharmaceutical and application procedure, precautions, it's risk and benefits. As a result, patient assures and actively participates in procedures.

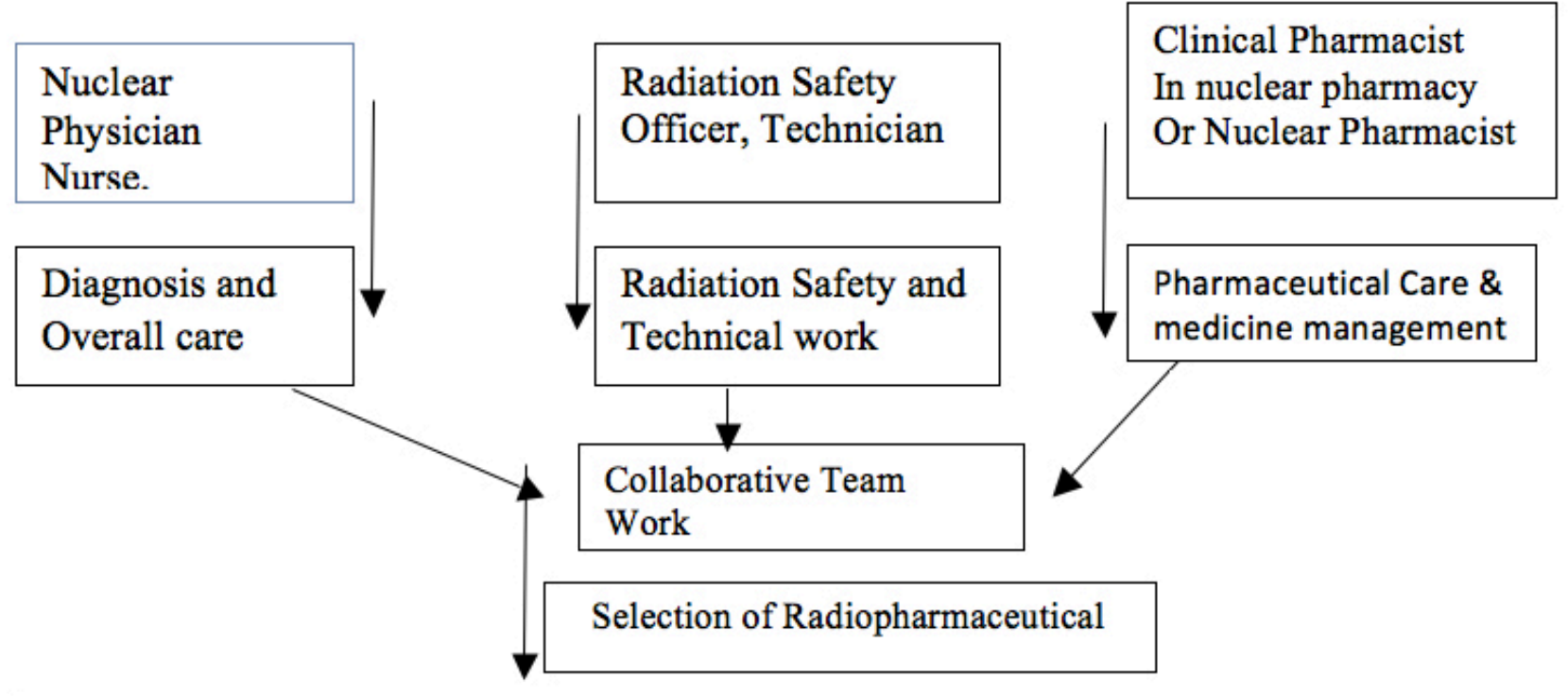

Prepare patient for procedure; prerequisite requirement and instruction, patient counseling

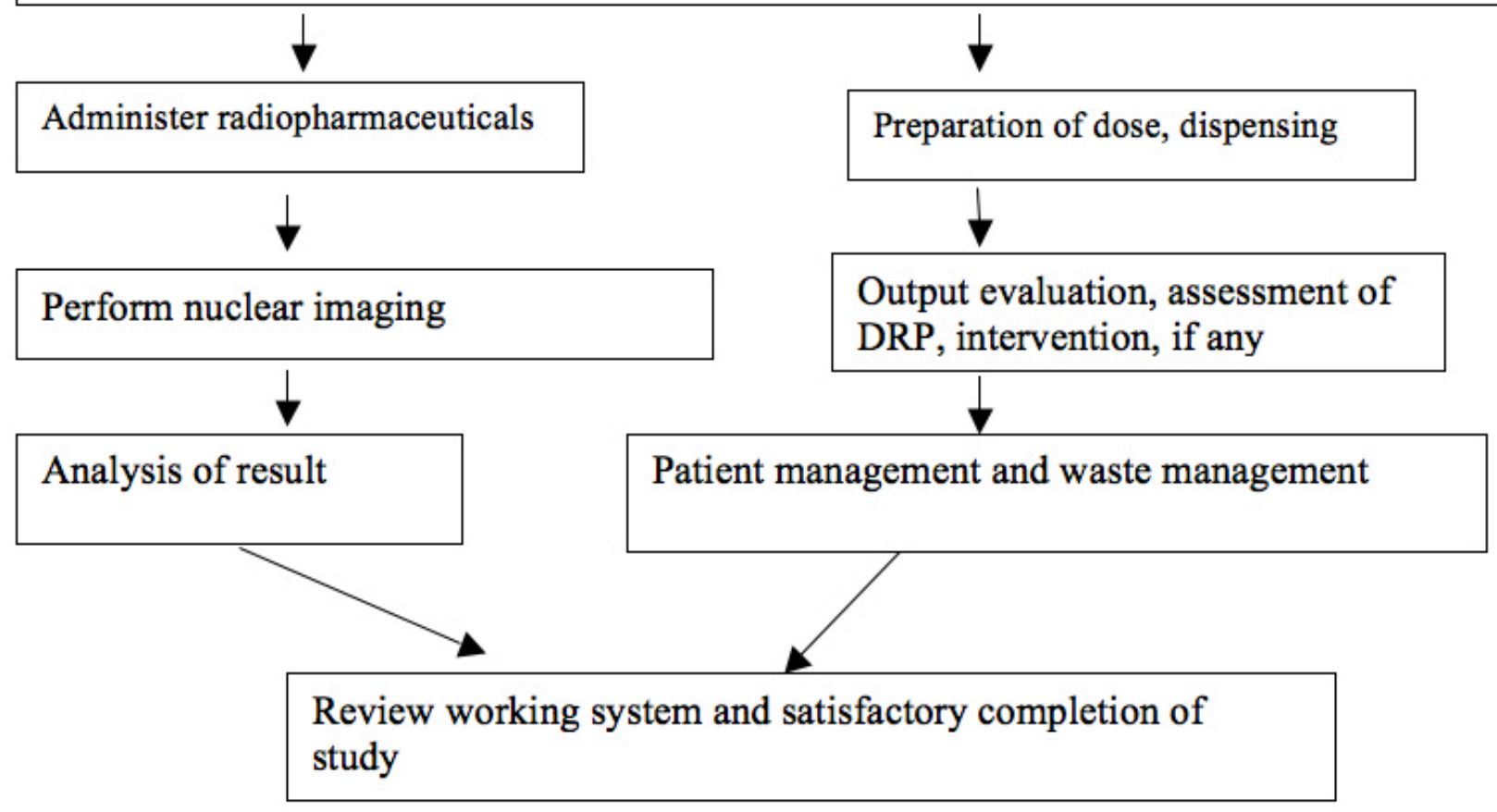

Figure 1: Activity of clinical pharmacist within healthcare at nuclear medicine department. 
After definition of pharmaceutical care as defined by Helper and Strand, Pharmaceutical care has become an important component of general pharmacy practice. In general, it is a practice in which the pharmacist takes responsibility for a patient's drug therapy needs for the purpose of positive patient outcomes. ${ }^{12}$ Over the sphere, the specialty of nuclear pharmacy deals primarily with imaginations and diagnostic radiopharmaceuticals. As a result, it may appear to fall outside the precepts of pharmaceutical care than the pharmaceutical care performed in other medical specialty. However, when viewed more broadly, many activities routinely performed by nuclear pharmacists directly or indirectly contribute to positive patient outcomes.

A pharmaceutical care plan explains all of the responsibilities and functions to take in consideration to optimize-rationalized use of medicine to patient. It also includes identification of drug related problems and its resolutions. Wide variety of activity is possible in radio and nuclear pharmacy department. In order to provide optimal pharmaceutical care, a pharmacist must be enough knowledgeable and armed with skills and applied principle of clinical services and radiopharmaceuticals. (S) He must be enough competent to work by rendering routine clinical services because (s) he will be responsible for any cause. This responsibility is significant irrespective of diagnostic or therapeutic application of radiopharmaceuticals. In other mean, clinical pharmacy services in nuclear medicine is more attentive than the other medicine. Due to unique applications, use and strict regulatory guideline, eagle eyes are essential needs while dealing radiopharmaceuticals.

(Table 1) lists the clinical services that the clinical pharmacist can provide and thus may be a part of health care team. Clinical pharmacy is gaining wide acceptance in our settings though it takes time. It is essential that radio and nuclear pharmacy must be a part of clinical pharmacy to improve medicine use and patient care. Another changes has been started in present time is change in prices of medicine in such case how cost effectively manage by policy makers. Rapidly changing cost, and thus budget, lead to look forward for cost control. Pendulum of cost verses time factor is swinging from low to high and high to low. Therefore, services by clinical pharmacy may become an option.

\section{Table 1: Lists of the services that the clinical pharmacist perform}

Referral services

Conducts and review medication history;

Patient counselling

Compounding

Dose calculation and prepare ancillaries

Quality assurance

Part of health care team

Response monitoring

Prepare patient information leaflet and informative brochures

Prepare SOP

Regulatory compliance

Health and safety

Procurement of drugs and quality assurance

Administrative functions as per post and profile
Selection and advise patient against radio and nuclear referral

Determine potential factor that affects result and procedures, delay-early in result, alter pharmacokinetic and pharmacodynamics of medicines; assure patient-especially depressed, excited, disable patients, patient with compromised organ functions by providing entire process overview; review the use of OTC, herbal and use of any medicines; that may alter result/outcome if so report to treating radiologist - intervention for discontinuations and suggest alternative

Aware patient on significance of procedures, cautions, stabilization, movement and disposal of excretes, pre-administrative instruction viz: nil by mouth, with health food, waiting period after injection/administration,

Generator elution, kit reconstitution, preparation of products and other radiolabeling procedures

Calculate dose as per need, look at medicine factor and patient specific factors, arrange ancillaries viz: syringes, preparation table/counter/lead coat

Functional checks of instruments, equipment and devices and determination of radiopharmaceutical quality and purity (e.g., radionuclidic purity, radiochemical purity, chemical purity, particle size, sterility, apyrogenicity, etc.). quality assurance of clinical pharmacy services

Prepare medicine and dispense, carefully observe drug administration and imaging process,. Troubleshot pertaining to medicine, report drug related problem if any

Monitor and evaluate response, monitor patient for any Adverse drug reaction or drug related problems.

Direct and indirect way of patient education, awareness activity,

Prepare standard operating guideline if needed,

Undergoes professional development, prepare, report and quality assurance of activity, medicine use evaluation,

Radiation protection and proper handling of hazardous chemicals and biological specimens.

Procurement includes the ordering, receipt, storage and inventory control of radiopharmaceuticals, ancillary drugs, supplies and related materials. 


\section{CONCLUSION}

At present, clinical pharmacy services in nuclear medicines are in infancy. To work inside the radio and nuclear pharmacy, directives and policy find essential for future. It will be essential for the pharmacist that pharmacist realize their importance and accept their responsibilities. It will also be important for policy makers, health care professionals, and educational institutes understand potential of pharmacy as a health care provider to prepare student pharmacist, to allow them and to make them responsible for their valuable services like nuclear pharmacy. We wish development of nuclear pharmacy as specialty in coming year.

\section{ACKNOWLEDGEMENT}

The authors are indebted to Dr.RakeshVyas, Dr. Swati, Omprakash Sinha, and Gujarat Cancer Research Institute, Ahmedabad, Gujarat for allowing us to carry out project and for valuable suggestions.

\section{CONFLICTS OF INTEREST}

Nill

\section{REFERENCES}

1. Patidar AK, Patidar P, Tandel TS, Mobiya A, Selvam G, Jeyakandan M. Current Trends IN Nuclear Pharmacy Practice. International Journal of Pharmaceutical Sciences Review and Research. 2010; 5(2): 145-51.

2. Hariharan S, Sankar V, RAmanathan M. The road ahead for the PharmD programme in India. Introduction of PharmD. in India. Pharma Times. 2012; 44(9): 17-9.

3. Govt. of INDIA. ADVERTISEMENT NO. 01/2013(R-II), Available from URL: http://barc.gov.in.

4. Vikrant Verma. Nuclear Pharmacy: an updated review. IJPRD. 2011; 3(6): 218-25.

5. Kavula MP, Barnett CW. Job Satisfaction, positive practice aspects, work activities and personality characteristics of nuclear chain pharmacists. J Nucl Med Technol. 1990; 18: 270-4

6. Ponto JA. Satisfaction with board certification in nuclear pharmacy. Am Pharm. 1989; 29: 16-8, 20.

7. Government of India. Bhabha Atomic Research Centre. Result of the interview conducted for the recruitment of Pharmacist B. Assess at URL: http://www.barc.gov.in/careers/result174.pdf

8. Kelly DV, Bishop L, young S, Hawboldt J, Phillips L, Keough TM. Pharmacist and Physicians view on collaborative practice. Can Pharm J. 2013; 146(4): 218-26.

9. Mehta BH, Snyder ME, Nikitas A. Developing collaborative relationships between pharmacists and other health professionals. J Am Pharm Assoc. 2011; 51(3): 332-8.

10. Rigby D. Collaboration between doctors and pharmacists in the community. Aust Prescr. 2010; 33: 191-3.

11. Makowsky MJ, Schindel TJ, Rosenthal M, Campbell K, Tsuyuki RT, Madill HM. Collaboration between pharmacists, physicians and nurse practitioners: A qualitative investigation of working relationships in the inpatient medical setting. Journal of Inter professional care. 2009; 23(2): 169-84.

12. Hepler CD, Strand LM. Opportunities and responsibilities in pharmaceutical care. Am J Hosp Pharm. 1990; 47(3): 533-43. 